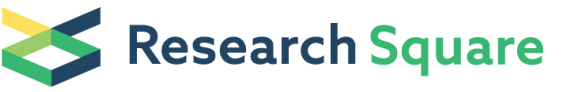 \\ Preprints are preliminary reports that have not undergone peer review. \\ They should not be considered conclusive, used to inform clinical practice, \\ or referenced by the media as validated information.
}

\section{Assessment The Burden of Recent Transmission of M. Tuberculosis Using WGS Analysis In Golmud City In China}

Chunfa Liu

National Center for Tuberculosis Control and Prevention, Chinese Center for Disease Control and Prevention

\section{Ping He}

National Institute for Communicable Disease Control and Prevention, Chinese Center for Disease Control and Prevention

Jiale Fan

National Center for Tuberculosis Control and Prevention, Chinese Center for Disease Control and Prevention

\section{Aijing Ma}

National Institute for Communicable Disease Control and Prevention, Chinese Center for Disease Control and Prevention

\section{Wencong He}

National Institute for Communicable Disease Control and Prevention, Chinese Center for Disease Control and Prevention

\section{Bing Zhao}

National Center for Tuberculosis Control and Prevention, Chinese Center for Disease Control and Prevention

\section{Shengfen Wang}

National Center for Tuberculosis Control and Prevention, Chinese Center for Disease Control and Prevention

\section{Guangxue He}

National Institute for Viral Diseases Control and Prevention, Chinese Center for Disease Control and Prevention

\section{Yanlin Zhao ( $\sim$ Zhaoyl@chinacdc.cn )}

National Center for Tuberculosis Control and Prevention, Chinese Center for Disease Control and Prevention

\section{Research Article}

Keywords: Tuberculosis, WGS, Recent transmissions, Risk factor, Drug resistant 
Posted Date: July 14th, 2021

DOI: https://doi.org/10.21203/rs.3.rs-668389/v1

License: (c) (i) This work is licensed under a Creative Commons Attribution 4.0 International License. Read Full License 


\section{Abstract}

Background: Whole genome based Mycobacterium tuberculosis (Mtb) surveillance is facilitated to tuberculosis control. The proportion of genotypic clusters in a population represents the recent transmission rate of Mtb.

Methods: We did a population based study of culture-positive Mtb in Golmud, Qinghai, China. Wholegenome sequencing (WGS) was used to discriminate the apparent genetic clusters and resistant associated genes of Mtb, and the risk of genomically clustered Mtb was analyzed combined with epidemiological data.

Results: A total of 133 cases of culture-positive tuberculosis were recorded between Jan 1, 2013 and Dec $31,2018,17(13 \%, 17 / 133)$ cases of which were multidrug-resistant/rifampicin tuberculosis (MDR/RRTB). Patients who were previous treatment or were younger than 35 years had high risk of MDR/RR-TB. $62(47 \%, 62 / 132)$ strains were in 23 genomic clusters that differed by 12 or fewer single nucleotide polymorphisms (SNPs), indicating recent transmission. Patients who were Tibetan nationality, or those $35-44$ years old were more likely to have recent transmission. $15(65 \%, 15 / 23)$ patients with genotypic rifampin resistant tuberculosis have epidemiological link. Mutation of rifampicin resistance associated genes in $r p o B$ Ser450Leu was showed lower cluster rate $(42 \%, 5 / 12)$ compared with other mutations.

Conclusions: Recent transmissions of Mtb strains, especially genotypic MDR/RR strains, drive the tuberculosis epidemic in Golmud, Qinghai, China.

\section{Background}

Tuberculosis (TB) is one of the top 10 causes of death and the leading cause of death among infectious diseases globally. China has the third largest tuberculosis epidemic and second highest MDR/RR-TB incidence worldwide, with an estimated 833000 new cases and 65000 MDR/RR-TB patients in 2019 [1]. The high TB burden, the high prevalence of MDR/RR-TB and high rate of transmission of drug resistant TB are still significant challenges which pose a major hurdle for TB control in our country [2, 3]. A combined analysis of 11 mathematical models in three high-burden countries has showed that full scaleup of all interventions in tuberculosis-programme performance fell short of the post-2015 End TB Strategy in China [4]. Transmission is the major driving force of the TB epidemic, especially, the MDR/RRTB pandemic [5]. To detect this spread, understand the dynamics of the disease, and develop optimized TB control strategies, accurate tracing of pathogen transmission in the host population is of outmost importance.

Molecular epidemiological methods that combine epidemiological investigations and genotyping of Mtb strains provide the ways to assess the recent transmission (within 2-3 years) of Mtb and risk factors for transmission [6]. Importantly, recent studies applying molecular strain typing indicated that transmission of MDR strains rather than insufficient treatment is one major driving force for the actual MDR epidemic [6-9]. WGS based genotyping has a better discriminatory power compared to classical genotyping, such 
as spoligotyping or MIRU-VNTR (Mycobacterial Interspersed Repetitive Units-Variable Number of Tandem Repeats) typing [10]. Furthermore, WGS provides a highly accurate identification of the diverse lineages of the Mtb complex and potentially allows for comprehensive detection of drug resistance mediating genomic variants [11]. A core genome multilocus sequence typing (cg-MLST) for Mtb complex strains based on a set of 2891 genes has been used to detection of epidemiologically linked tuberculosis cases, and a difference in more than 12 alleles of the scheme has been suggested as the threshold to rule out recent transmission $[12,13]$.

In China, only a few population-based studies systematically evaluated the contribution of recent transmission to the disease burden, and most of these studies were performed in more developed areas, e.g. Shanghai and Shenzhen city $[6,14,15]$. Few studies were evaluated the recent transmission burden in low population density and less developed areas. In this study, we did a population-based study to evaluate the contribution of recent transmission to the TB disease burden in Golmud city which is a large city with a small population, in Qinghai province of China. We analyzed the risk factors for transmission by using WGS and epidemiological investigations.

\section{Methods}

\section{Study design and population}

Golmud city is one of the 72 sites from the national survey of drug-resistant tuberculosis in China with an estimated population of 200 thousand [16]. All suspected pulmonary tuberculosis patients are referred to local designated hospitals, where the diagnosis is made by sputum smear and culture. Through a routine surveillance system, all cases of tuberculosis are reported to Qinghai Municipal Centre for Disease Control and Prevention (CDC) and all cultured positive samples are delivered to China CDC. This population-based, retrospective observational study included all patients aged 15 years or older with culture-confirmed pulmonary tuberculosis who were reported by local designated hospitals in Golmud between Jan 1, 2013, to Dec 31, 2018. The epidemiological investigation of demographic, sexual, educational, job-seeking characteristics and national information are collected from patients after written informed consent. The institutional review boards of China CDC approved the study.

\section{Procedures}

As we have showed [16], two sputum samples for culturing were obtained from each eligible patient before the initiation of treatment. For isolation of the culture, each specimen was treated with one volume of $4 \%$ sodium hydroxide per one volume of sputum and was then homogenized by vigorous stirring. An aliquot of $0.1 \mathrm{ml}$ of the resulting specimen was inoculated into two tubes of acidified LöwensteinJensen medium and incubated at $37^{\circ} \mathrm{C}$. The culture was assessed during week 1 for rapidly growing bacteria and again every week thereafter for slower growing bacteria; if no bacteria grew by 8 weeks, the result was recorded as negative. Cultures with growing colonies were sent to the national reference laboratory for tuberculosis (NTRL) in China CDC for identification and WGS analysis. Matrix-assisted laser desorption/ionization time-of-flight mass spectrometry (MADLI-TOF-MS) was used to differentiate 
Mycobacterium tuberculosis from other mycobacteria. We used the cetyl trimethyl ammonium bromide (CTAB) method to extract genomic DNA from cultures from one sputum specimen per patient. Wholegenome sequencing was done with the Illumina Hiseq 2500 (Illumina, San Diego, CA, USA) with an expected coverage of $100 \times$.

We used the functionality implemented in the Ridom SeqSphere + software (version 7.2.3) with default settings to perform cg-MLST analysis. The genome of the Mtb strain H37Rv (GenBank ID NC_00962.3) served as seed genome. Subsequently, obtained genomes were compared with the seed genome to establish a list of core genome genes. Here, default settings include the removal of the shorter of two genes overlapping by more than four bases and of genes with an internal stop codon in $>90 \%$ of all genomes from the scheme. Cg-MLST based neighbour joining and minimum spanning trees were both calculated and drawn with the SeqSphere + software.

The SNP-based approach was performed by excluding regions annotated as repetitive elements (e.g. PPE and PE-PGRS gene families), InDels, multiple consecutive SNPs in a 12-bp window (possible InDel artefacts or rare recombination scars). For the phylogenetic reconstruction the SNP frequency was set to $75 \%$ and a genome position was considered valid when $95 \%$ of the combined strains had enough coverage (four reads per direction) at this position.

Resistance genotypes were defined using the web-software: Mykrobe is available at: http://mykrobe.com. TB-Profiler v2.8.14 is available at: https://github.com/jodyphelan/TBProfiler [17]. NEJM-2018 approach was described as show in the published paper [18].

\section{Statistical analysis}

Descriptive statistics was performed for patients' demographics and lineages, resistance categories as well as clustering status of MTBC strains. Data derived from genomic analysis of Clinical isolates were analyzed statistically using IBM SPSS Statistics Software for Windows (version 19) and R (version 3.6.1). For univariate analysis of potential factors associated with pre-XDR/XDR TB we performed a fisher's exact test. Factors with a significant result in the univariate model were included into a multivariate logistic regression analysis. Odd ratios with $95 \%$ confidence interval $(\mathrm{Cl})$ were estimated and variables with $P$ values less than 0.05 were taken as significant predictors.

\section{Results}

As showed in Fig. 1, from Jan 1, 2013, to Dec 31, 2018, 343 people were diagnosed as tuberculosis in Golmud city, while 134 were diagnosed as culture-positive tuberculosis. 1 people were excluded as the strains species identification by MADLI-TOF-MS as NTM. Drug susceptibility test (DST) was performed in the remaining 133 individuals, 17 (12.78\%) were rifampicin (RIF) resistance, 16 (12.03\%) were isoniazide $(\mathrm{INH})$ resistance, $4(3.01 \%)$ were ethambutol (EMB) resistance. No strains were resistant to aminoglycoside drugs. Lower fluoroquinolones resistant rates were observed, 1 (0.75\%) was moxifloxacin (MOXF) resistance and 2 (1.50\%) were levofloxacin (LEVF) resistance as showed in Fig. 2A. From 2013 to 
2018, the resistant rate in INH and RIF was presented a decreasing tendency since 2016 as showed in Fig. 2B. This may attribute to the employ of phenotypic DST to provide appropriate treatment of drugresistant tuberculosis. As showed in Table 1, the patients with MDR/RR-TB were significantly younger (age $15-34$ years vs $45-64, P=0.03$ by univariate analysis, $P=0.01$ by multivariate analysis), and were more likely to occur in previously treated patients (35\% vs $8 \%, P=0.002$ by univariate analysis, $P=0.0001$ by multivariate analysis). 
Table 1

Univariable and multivariable logistic regression on the risk factors of MDR/RR-TB

\begin{tabular}{|c|c|c|c|c|c|c|}
\hline Factors & $\begin{array}{l}\text { Non- } \\
\text { RR/MDR-TB }\end{array}$ & $\begin{array}{l}\text { RR/MDR- } \\
\text { TB }\end{array}$ & OR $(95 \% \mathrm{Cl})$ & $\begin{array}{l}\mathbf{p} \\
\text { value }\end{array}$ & $\begin{array}{l}\text { Adjusted OR } \\
(95 \% \mathrm{Cl})\end{array}$ & $\begin{array}{l}p \\
\text { value }\end{array}$ \\
\hline \multicolumn{7}{|l|}{ Sex } \\
\hline Female & 50 (43\%) & 5 (29\%) & 1.00 & & 1.00 & \\
\hline Male & $66(57 \%)$ & $12(71 \%)$ & $\begin{array}{l}1.82(0.60- \\
5.50)\end{array}$ & 0.29 & $\begin{array}{l}0.34(0.06- \\
1.85)\end{array}$ & 0.21 \\
\hline \multicolumn{7}{|l|}{ Age, year } \\
\hline$\geq 65$ & 18 (16\%) & $1(6 \%)$ & $\begin{array}{l}1.83(0.11- \\
31.09)\end{array}$ & 0.68 & $\begin{array}{l}3.59(0.07- \\
174.12)\end{array}$ & 0.52 \\
\hline $45-64$ & 33 (29\%) & $1(6 \%)$ & 1.00 & & 1.00 & \\
\hline $35-44$ & 24 (21\%) & $3(18 \%)$ & $\begin{array}{l}4.13(0.40- \\
42.12)\end{array}$ & 0.23 & $\begin{array}{l}26.79(0.58- \\
1248.91)\end{array}$ & 0.09 \\
\hline $15-34$ & 40 (35\%) & 12 (71\%) & $\begin{array}{l}9.90(1.22- \\
80.15)\end{array}$ & 0.03 & $\begin{array}{l}231.60(4.18- \\
12824.62)\end{array}$ & 0.01 \\
\hline \multicolumn{7}{|l|}{ Residence } \\
\hline Urban & 39 (34\%) & $4(24 \%)$ & 1.00 & & & \\
\hline Rural & 76 (66\%) & 13 (76\%) & $\begin{array}{l}1.67(0.51- \\
5.46)\end{array}$ & 0.40 & $\begin{array}{l}7.46(0.61- \\
90.83)\end{array}$ & 0.12 \\
\hline \multicolumn{7}{|l|}{ Occupation } \\
\hline Farmer & 76 (66\%) & 10 (59\%) & 1.00 & & 1.00 & \\
\hline Worker & 18 (16\%) & $4(24 \%)$ & $\begin{array}{l}1.79(0.50- \\
6.39)\end{array}$ & 0.37 & $\begin{array}{l}2.50(0.17- \\
37.96)\end{array}$ & 0.51 \\
\hline Student & $2(2 \%)$ & $1(6 \%)$ & $\begin{array}{l}3.80(0.32- \\
45.80)\end{array}$ & 0.29 & $\begin{array}{l}5.17(0.16- \\
166.27)\end{array}$ & 0.35 \\
\hline Others & $19(17 \%)$ & $2(12 \%)$ & $\begin{array}{l}0.76(0.15- \\
3.75)\end{array}$ & 0.74 & $\begin{array}{l}0.17(0.01- \\
2.42)\end{array}$ & 0.19 \\
\hline \multicolumn{7}{|l|}{ Nationality } \\
\hline Han & 45 (39\%) & $4(24 \%)$ & 1.00 & & 1.00 & \\
\hline Mongolian & $7(6 \%)$ & $3(18 \%)$ & $\begin{array}{l}4.82(0.89- \\
26.28)\end{array}$ & 0.07 & $\begin{array}{l}1.74(0.05- \\
57.59)\end{array}$ & 0.76 \\
\hline Hui & $25(22 \%)$ & $4(24 \%)$ & $\begin{array}{l}1.80(0.41- \\
7.83)\end{array}$ & 0.43 & $\begin{array}{l}1.37(0.10- \\
18.07)\end{array}$ & 0.81 \\
\hline Tibetan & 37 (32\%) & $6(35 \%)$ & $\begin{array}{l}1.82(0.48- \\
6.95)\end{array}$ & 0.38 & $\begin{array}{l}0.70(0.06- \\
8.09)\end{array}$ & 0.78 \\
\hline
\end{tabular}




\begin{tabular}{|c|c|c|c|c|c|c|}
\hline Factors & $\begin{array}{l}\text { Non- } \\
\text { RR/MDR-TB }\end{array}$ & $\begin{array}{l}\text { RR/MDR- } \\
\text { TB }\end{array}$ & OR (95\% Cl) & $\begin{array}{l}\mathrm{p} \\
\text { value }\end{array}$ & $\begin{array}{l}\text { Adjusted OR } \\
\text { (95\% Cl) }\end{array}$ & $\begin{array}{l}\mathrm{p} \\
\text { value }\end{array}$ \\
\hline Uyghur & $2(2 \%)$ & 0 & - & - & - & - \\
\hline \multicolumn{7}{|l|}{$\begin{array}{l}\text { Previous } \\
\text { treatment }\end{array}$} \\
\hline No & 107 (92\%) & $11(65 \%)$ & 1.00 & & 1.00 & \\
\hline Yes & $9(8 \%)$ & $6(35 \%)$ & $\begin{array}{l}6.49(1.94- \\
21.64)\end{array}$ & 0.002 & $\begin{array}{l}163.83(11.21- \\
2393.85)\end{array}$ & 0.0001 \\
\hline \multicolumn{7}{|l|}{ Educational level } \\
\hline $\begin{array}{l}\text { Collage or above } \\
\text { degree }\end{array}$ & $6(5 \%)$ & $2(12 \%)$ & $\begin{array}{l}3.67(0.27- \\
49.29)\end{array}$ & 0.33 & $\begin{array}{l}3.70(0.04- \\
318.59)\end{array}$ & 0.57 \\
\hline $\begin{array}{l}\text { Senior high } \\
\text { degree }\end{array}$ & $11(9 \%)$ & $1(6 \%)$ & 1.00 & & 1.00 & \\
\hline $\begin{array}{l}\text { Junior high } \\
\text { degree }\end{array}$ & 31 (27\%) & $4(24 \%)$ & $\begin{array}{l}1.42(0.14- \\
14.11)\end{array}$ & 0.77 & $\begin{array}{l}4.11(0.06- \\
285.02)\end{array}$ & 0.51 \\
\hline Primary degree & 31 (27\%) & $4(24 \%)$ & $\begin{array}{l}1.42(0.14- \\
14.11)\end{array}$ & 0.77 & $\begin{array}{l}21.30(0.27- \\
1672.39)\end{array}$ & 0.17 \\
\hline Other & 37 (32\%) & $6(35 \%)$ & $\begin{array}{l}1.78(0.19- \\
16.45)\end{array}$ & 0.61 & $\begin{array}{l}59.94(0.48- \\
7548.97)\end{array}$ & 0.10 \\
\hline \multicolumn{7}{|l|}{ TB contact } \\
\hline Yes & $15(13 \%)$ & $2(12 \%)$ & 1.00 & & 1.00 & \\
\hline No & $101(87 \%)$ & 15 (88\%) & $\begin{array}{l}1.11(0.23- \\
5.36)\end{array}$ & 0.89 & $\begin{array}{l}6.67(0.42- \\
106.11)\end{array}$ & 0.18 \\
\hline \multicolumn{7}{|l|}{ Income } \\
\hline$\leq 10000$ & 64 (56\%) & $14(82 \%)$ & 1.00 & & 1.00 & \\
\hline $10000-50000$ & $46(40 \%)$ & $3(18 \%)$ & $\begin{array}{l}0.30(0.08- \\
1.10)\end{array}$ & 0.07 & $\begin{array}{l}0.37(0.05- \\
2.90)\end{array}$ & 0.34 \\
\hline$>50000$ & $5(4 \%)$ & 0 & - & - & - & - \\
\hline \multicolumn{7}{|l|}{ Lineage } \\
\hline Lineage 2 & 107 & 16 & 1.00 & & 1.00 & \\
\hline Lineage 4 & 8 & 1 & $\begin{array}{l}0.84(0.10- \\
7.14)\end{array}$ & 0.87 & $\begin{array}{l}1.76(0.04- \\
79.04)\end{array}$ & 0.77 \\
\hline
\end{tabular}

Of the 132 culture-positive individuals for whom sufficient WGS data were available, 62 (47\%, 62/132) strains were in 23 genomic clusters that differed by 12 or fewer SNPs which presented recent transmission based on the cg-MLST approach, as showed in Fig. 3A. To increase accuracy in identifying TB transmission clusters, a SNP-based pipeline was applied to analyze. As showed in Fig. 3B, 60 strains 
were classified into 22 clusters that same with cg-MLST approach. The SNP distance of the remaining two strains is 13 that close to the 12 SNP thresholds. Of all the 62 strains, $81.82 \%$ have the diagnosis interval less than 3 years, as showed in Fig. 4. This may imply most of the recent transmission issues were happened within 3 or less years. Table 2 shows the analysis results of risk factors between clustered or non-clustered strains; the recent transmission was more occurred in patients who are 35-44 years old compared with $15-34$ years old ( $P=0.05$ by univariate analysis), farmer compared with worker $(P=0.05$ by univariate analysis), or Tibetan compared with Han nationality ( $P=0.02$ by univariate analysis). Multivariate analysis indicated that recent transmission of TB were more happened in patients with Tibetan nationality $(P=0.02)$ or elder age (35-44 or $\geq 65$ years, $P=0.01$ and $P=0.04$ ). 
Table 2

Univariable and multivariate logistic regression on the risk factors of Mtb clustering $(n=132)$

\begin{tabular}{|c|c|c|c|c|c|c|}
\hline Factors & $\begin{array}{l}\text { Non- } \\
\text { cluster }\end{array}$ & Cluster & OR((95\% Cl)) & value & $\begin{array}{l}\text { Adjusted OR (95\% } \\
\text { Cl) }\end{array}$ & $\begin{array}{l}\mathrm{P}- \\
\text { value }\end{array}$ \\
\hline \multicolumn{7}{|l|}{ Sex } \\
\hline Female & $31(44 \%)$ & $\begin{array}{l}23 \\
(37 \%)\end{array}$ & 1.00 & & 1.00 & \\
\hline Male & $39(56 \%)$ & $\begin{array}{l}39 \\
(63 \%)\end{array}$ & $\begin{array}{l}1.35(0.67- \\
2.71)\end{array}$ & 0.40 & $0.93(0.38-2.27)$ & 0.88 \\
\hline \multicolumn{7}{|l|}{ Age, year } \\
\hline $15-34$ & $31(44 \%)$ & $\begin{array}{l}20 \\
(33 \%)\end{array}$ & 1.00 & & 1.00 & \\
\hline $35-44$ & $\begin{array}{l}10 \\
(14 \%))\end{array}$ & $\begin{array}{l}17 \\
(28 \%)\end{array}$ & $\begin{array}{l}2.64(1.01- \\
6.90)\end{array}$ & 0.05 & $\begin{array}{l}5.24(1.44- \\
19.03)\end{array}$ & 0.01 \\
\hline $45-64$ & $19(27 \%)$ & $\begin{array}{l}15 \\
(25 \%)\end{array}$ & $\begin{array}{l}1.22(0.51- \\
2.95)\end{array}$ & 0.65 & $2.15(0.64-7.22)$ & 0.22 \\
\hline$\geq 65$ & $10(14 \%)$ & $\begin{array}{l}9 \\
(15 \%)\end{array}$ & $\begin{array}{l}1.40(0.48- \\
4.03)\end{array}$ & 0.54 & $\begin{array}{l}4.57(1.11- \\
18.87)\end{array}$ & 0.04 \\
\hline \multicolumn{7}{|l|}{ Residence } \\
\hline Urban & 27 (39\%) & $\begin{array}{l}16 \\
(26 \%)\end{array}$ & 1.00 & & 1.00 & \\
\hline Rural & $43(61 \%)$ & $\begin{array}{l}45 \\
(74 \%)\end{array}$ & $\begin{array}{l}1.77(0.84- \\
3.73)\end{array}$ & 0.14 & $1.23(0.44-3.42)$ & 0.70 \\
\hline \multicolumn{7}{|l|}{ Occupation } \\
\hline Worker & $16(23 \%)$ & $\begin{array}{l}6 \\
(10 \%)\end{array}$ & 1.00 & & 1.00 & 1.00 \\
\hline Farmer & $42(60 \%)$ & $\begin{array}{l}44 \\
(72 \%)\end{array}$ & $\begin{array}{l}2.79(1.00- \\
7.82)\end{array}$ & 0.05 & $\begin{array}{l}2.54(0.48- \\
13.32)\end{array}$ & 0.27 \\
\hline Student & $3(4 \%)$ & 0 & - & - & - & - \\
\hline Others & $9(13 \%)$ & $\begin{array}{l}11 \\
(18 \%)\end{array}$ & $\begin{array}{l}3.26(0.90- \\
11.81)\end{array}$ & 0.72 & $\begin{array}{l}3.23(0.54- \\
19.35)\end{array}$ & 0.20 \\
\hline \multicolumn{7}{|l|}{ Nationality } \\
\hline Han & $30(43 \%)$ & $\begin{array}{l}19 \\
(31 \%)\end{array}$ & 1.00 & & 1.00 & \\
\hline Mongolian & $7(10 \%)$ & $3(5 \%)$ & $\begin{array}{l}0.68(0.16- \\
2.94)\end{array}$ & 0.60 & $1.71(0.20-14.54)$ & 0.63 \\
\hline
\end{tabular}




\begin{tabular}{|c|c|c|c|c|c|c|}
\hline Factors & $\begin{array}{l}\text { Non- } \\
\text { cluster }\end{array}$ & Cluster & OR((95\% Cl)) & $\mathrm{p}_{\text {value }}$ & $\begin{array}{l}\text { Adjusted OR (95\% } \\
\text { Cl) }\end{array}$ & $\begin{array}{l}\mathrm{P} \text { - } \\
\text { value }\end{array}$ \\
\hline Hui & $17(24 \%)$ & $\begin{array}{l}12 \\
(19 \%)\end{array}$ & $\begin{array}{l}1.12(0.44- \\
2.84)\end{array}$ & 0.82 & $1.55(0.44-5.12)$ & 0.50 \\
\hline Tibetan & $15(21 \%)$ & $\begin{array}{l}27 \\
(44 \%)\end{array}$ & $\begin{array}{l}2.57(1.10- \\
5.99)\end{array}$ & 0.02 & $\begin{array}{l}5.23(1.32- \\
20.73)\end{array}$ & 0.02 \\
\hline Uyghur & $1(1 \%)$ & $1(2 \%)$ & $\begin{array}{l}1.58(0.09- \\
26.78)\end{array}$ & 0.75 & $\begin{array}{l}11.05(0.31- \\
395.29)\end{array}$ & 0.19 \\
\hline \multicolumn{7}{|l|}{ Previous treatment } \\
\hline No & $63(90 \%)$ & $\begin{array}{l}55 \\
(89 \%)\end{array}$ & 1.00 & & 1.00 & \\
\hline Yes & $7(10 \%)$ & $\begin{array}{l}7 \\
(11 \%)\end{array}$ & $\begin{array}{l}1.15(0.38- \\
3.47)\end{array}$ & 0.81 & $\begin{array}{l}2.79(0.59- \\
13.21)\end{array}$ & 0.20 \\
\hline \multicolumn{7}{|l|}{ Educational level } \\
\hline $\begin{array}{l}\text { Collage or above } \\
\text { degree }\end{array}$ & $6(9 \%)$ & $2(3 \%)$ & 1.00 & & 1.00 & \\
\hline Senior high degree & $7(10 \%)$ & $5(8 \%)$ & $\begin{array}{l}2.14(0.30- \\
15.36)\end{array}$ & 0.45 & $0.42(0.04-4.45)$ & 0.47 \\
\hline Junior high degree & $17(24 \%)$ & $\begin{array}{l}18 \\
(29 \%)\end{array}$ & $\begin{array}{l}3.18(0.56- \\
17.96)\end{array}$ & 0.19 & $0.26(0.02-2.85)$ & 0.27 \\
\hline Primary degree & $19(27 \%)$ & $\begin{array}{l}15 \\
(24 \%)\end{array}$ & $\begin{array}{l}2.37(0.42- \\
13.46)\end{array}$ & 0.33 & $0.20(0.02-2.27)$ & 0.20 \\
\hline Other & $21(30 \%)$ & $\begin{array}{l}22 \\
(35 \%)\end{array}$ & $\begin{array}{l}3.14(0.57- \\
17.35)\end{array}$ & 0.19 & $0.11(0.01-2.27)$ & 0.10 \\
\hline \multicolumn{7}{|l|}{ TB contact } \\
\hline Yes & $9(13 \%)$ & $\begin{array}{l}8 \\
(13 \%)\end{array}$ & 1.00 & & 1.00 & \\
\hline No & $61(87 \%)$ & $\begin{array}{l}54 \\
(87 \%)\end{array}$ & $\begin{array}{l}1.00(0.36- \\
2.76)\end{array}$ & 0.99 & $1.42(0.40-5.05)$ & 0.59 \\
\hline \multicolumn{7}{|l|}{ Income } \\
\hline$\leq 10000$ & $41(59 \%)$ & $\begin{array}{l}36 \\
(59 \%)\end{array}$ & 1.00 & & 1.00 & \\
\hline $10000-50000$ & $28(40 \%)$ & $\begin{array}{l}21 \\
(34 \%)\end{array}$ & $\begin{array}{l}0.85(0.42- \\
1.76)\end{array}$ & 0.67 & $1.22(0.46-3.26)$ & 0.69 \\
\hline$>50000$ & $1(1 \%)$ & $4(7 \%)$ & $\begin{array}{l}4.56(0.49- \\
42.65)\end{array}$ & 0.15 & $\begin{array}{l}7.93(0.65- \\
96.50)\end{array}$ & 0.10 \\
\hline
\end{tabular}




\begin{tabular}{|lllllll|}
\hline Factors & $\begin{array}{l}\text { Non- } \\
\text { cluster }\end{array}$ & Cluster & OR((95\% Cl)) & $\begin{array}{l}\text { p } \\
\text { value }\end{array}$ & $\begin{array}{l}\text { Adjusted OR (95\% } \\
\text { Cl) }\end{array}$ & $\begin{array}{l}\text { P- } \\
\text { value }\end{array}$ \\
\hline No & $62(89 \%)$ & $\begin{array}{l}47 \\
(76 \%)\end{array}$ & 1.00 & & 1.00 & \\
\hline Yes & $8(11 \%)$ & $\begin{array}{l}15 \\
(24 \%)\end{array}$ & $\begin{array}{l}2.47(0.97- \\
6.32)\end{array}$ & 0.06 & $\begin{array}{l}7.86(1.87- \\
33.01)\end{array}$ & 0.01 \\
\hline Lineage & & & & & & \\
\hline Lineage 4 & $7(10 \%)$ & $2(3 \%)$ & 1.00 & & 1.00 & 0.16 \\
\hline Lineage 2 & $63(90 \%)$ & $\begin{array}{l}60 \\
(97 \%)\end{array}$ & $\begin{array}{l}3.33(0.67- \\
16.69)\end{array}$ & 0.14 & $\begin{array}{l}4.58(0.56- \\
37.33)\end{array}$ & \\
\hline
\end{tabular}

Using phenotypic DST as gold standard, we measured performance characteristics of WGS-based DST, including sensitivity and specificity. As showed in Table 3, the three tools for detection of resistanceassociated alleles showed similar sensitivity rate and specificity rate. The difference in TB-profiler and Mykrobe or NEJM-2018 for INH and RIF were attributed to the lack of mutations of rpoB D545E and katG G125D in TB-profiler datasets. Of all 132 strains, 9 were lineage 4 and 113 were lineage 2 identified by TB-profiler and Mykrobe software. In Fig. 5, we observed an interesting phenomenon that the strains with drug resistant mutations were clustered in phylogenetic tree. Then we tested if the strains with RIF associated resistant mutations were more likely to have recent transmission. As showed in Table 2, the patients $(65 \%, 15 / 23)$ who infected by RIF genotypic resistance tuberculosis are more likely to occur cluster transmission ( $P=0.01$ by multivariate analysis). The results implied that transmission of genotypic MDR/RR strains accounted for most $(57 \%, 13 / 23)$ cases of genotypic MDR/RR-TB overall. Then we analyzed the cluster rate based on mutation sites of rpoB gene, as showed in Fig. 6A,Ser450Leu was the most common mutation site. As showed in Fig. 6B, the cluster rate of each RIF genotypic resistant strains showed correlation with phenotypic resistant detection rate. A significantly negative correlation between cluster rate and phenotypic resistant detection rate among all 23 RIF genotypic resistant strains based on each mutation site was confirmed with a correlation coefficient $R=-0.9432$, as showed in Fig. 6C. 
Table 3

WGS compared with phenotypic DST in drug resistance diagnosis of Mtb

\begin{tabular}{|lllllll|}
\hline Drugs & TB-Profiler & \multicolumn{3}{c|}{ Mykrobe } & \multicolumn{3}{c|}{ NEJM-2018 } \\
\cline { 2 - 6 } & Sensitivity & Specificity & Sensitivity & Specificity & Sensitivity & Specificity \\
\hline INH & $81.25 \%$ & $95.69 \%$ & $81.25 \%$ & $94.83 \%$ & $81.25 \%$ & $94.83 \%$ \\
\hline RIF & $94.12 \%$ & $93.91 \%$ & $94.12 \%$ & $93.04 \%$ & $94.12 \%$ & $93.04 \%$ \\
\hline EMB & $75.00 \%$ & $98.44 \%$ & $75.00 \%$ & $98.44 \%$ & $75.00 \%$ & $98.44 \%$ \\
\hline KAN & - & $100 \%$ & - & $100 \%$ & - & $100 \%$ \\
\hline AMI & - & $100 \%$ & - & $100 \%$ & - & $100 \%$ \\
\hline LEVF & $100 \%$ & $99.23 \%$ & $50 \%$ & $100 \%$ & $50 \%$ & $100 \%$ \\
\hline MOXF & $100 \%$ & $98.47 \%$ & $100 \%$ & $100 \%$ & $100 \%$ & $100 \%$ \\
\hline
\end{tabular}

\section{Discussion}

In our study, we analyzed the recent transmission on TB burden by WGS in Golmud city of China. Recent transmission was happened in $47 \%$ patients with culture-positive TB; individuals who are Tibetan nationality or elder age were more likely to happen. We also found that $57 \%$ genotypic MDR/RR strains are clustered within 12 SNPs distance. Recent transmission, especially in genotypic MDR/RR-TB, drives the tuberculosis epidemic in Golmud, Qinghai, China.

The traditional epidemiological method for detecting transmission networks of TB patients mainly is based on interviews [19]. As a gold standard of transmission linking, this method does not always match the true transmission patterns [7]. Molecular epidemiology is a genotyping method that has been used to Mtb strains for determining whether two or more patients are linked within a transmission chain. The WGS-based methods for analysis Mtb strains cluster determination has been proved to have high discriminatory power when assessing transmission dynamics [20-22], either using cg-MLST [5, 12, 21, 23] or SNP distances [24, 25]. In our research, we compared the cg-MLST approach and a SNP-based pipeline to detect epidemiologically linked TB cases. The two approaches got same results, except two strains in cluster 23 defined by cg-MLST showed a 13 SNPs distance by SNP-based pipeline. SNP and cgMLST based analyses have similar clustering characteristics for data obtained from a high incidence setting [21].

'Recent transmission' is often applied to gain a better understanding of the current transmission dynamics of pathogens in a given population [21]. Genetic distance below 5-12 SNPs can be used as threshold to explain the recent transmission events. As a high TB incidence country, 12 SNPs distance was always used in China [14, 26]. 47\% patients with culture-positive TB were clustered, indicating recent transmission is a noticeable cause of TB patients in Golmud, China. The risk factors analysis indicated 
that patients with Tibetan nationality or elder age were higher transmission incidence. As a research in Shanghai city, China, older than 45 years had high risk of recent [6]. In China, older people should paid more attention on TB infection by transmission. Publicity about the TB transmission in rural areas needs to be improved.

MDR/RR-TB has emerged as a global threat and occurs more frequently in previously treated cases than in untreated patients. China has the second highest incidence of MDR/RR-TB, accounted for $14 \%$ of global MDR/RR-TB patients[1]. A meta-analysis about risk factors of MDR-TB in China showed that Han ethnic group, history of tuberculosis treatment, pulmonary cavity, migrant population, TB case contact history, living in rural areas and low income were considered to be at risk of MDR-TB [27, 28]. In Golmud city, 40\% previously treated cases are MDR/RR-TB. Effective TB control relies on early diagnosis and subsequent rapid DST to provide appropriate treatment in order to interrupt further transmission [29]. Since 2013, phenotypic DST was used and generated an increase of RIF and INH resistant rates, then following a sharp decline from 2016. Besides an improvement of treatment outcome of MDR-TB [29], a rapid DST test also decreased the transmission dynamics of phenotypic MDR/RR-TB. As an interesting phenomenon, genetic MDR/RR-TB based on WGS analysis had a high cluster rate and drove transmission dynamics in Golmud city. We considered that this may attribute to the consistency bias between phenotypic and genetic results. Some research has indicated that WGS more accurately predicts susceptibility of Mtb to first-line drugs than phenotypic testing [30]. Some genetic resistant strains were missed by phenotypic identification methods that lead the transmission of missing strains. Therefore, we agree the WGS and rapid testing methods will be the future of TB resistance diagnosis [31].

Our study has noteworthy limitations. First, the study time horizon makes it impossible that we collate all the tuberculosis cases and strains in the population. Second, strains could be misclassified as unique if they were in fact clustered with strains outside the study period and geographical setting. Third, the field epidemiologic investigation was insufficient that have missed some transmission settings and epidemiological links. In any case, WGS based epidemiologic analysis and drug resistant prediction supply us more advantageous information that will facilitate TB control whole world wide.

\section{Conclusions}

In conclusion, patients who were previous treatment or were younger than 35 years had high risk of MDR/RR-TB in Golmud, Qinghai, China. WGS analysis indicated that recent transmission of Mtb strains, especially genotypic MDR/RR-TB strains, drive the tuberculosis epidemic. Patients who were Tibetan nationality, or those 35-44 years old were more likely to have recent transmission. The low detection efficiency of strains with rifampicin resistant associated mutation by phenotypic drug susceptibility test methods may lead to the transmission.

\section{Abbreviations}

Mycobacterium tuberculosis: Mtb 
Whole-genome sequencing: WGS

Multidrug-resistant/rifampicin tuberculosis: MDR/RR-TB

Single nucleotide polymorphisms: SNPs

Tuberculosis: TB

Mycobacterial Interspersed Repetitive Units-Variable Number of Tandem Repeats: MIRU-VNTR

Core genome multilocus sequence typing: cg-MLST

Centre for Disease Control and Prevention: CDC

National reference laboratory for tuberculosis: NTRL

Matrix-assisted laser desorption/ionization time-of-flight mass spectrometry: MADLI-TOF-MS

Cetyl trimethyl ammonium bromide: CTAB

Drug susceptibility test: DST

Rifampicin: RIF

Isoniazide: INH

Ethambutol: EMB

Moxifloxacin: MOXF

Levofloxacin: LEVF

\section{Declarations}

\section{Ethics approval and consent to participate}

This research has been granted by the Ethics Committee of Chinese center for disease control and prevention, and an informed consent was obtained from each participant/respondent. All methods were carried out in accordance with relevant guidelines and regulations.

\section{Consent for publication}

This research including all the paper detail, data, tables and images was consented by all the authors and respondents in this paper for publication. And they will be freely available on the internet.

\section{Availability of data and materials}


Data supporting the results can be found in this paper. The datasets generated during and analyzed during the current study are available from the corresponding author zhaoyl@chinacdc.cn (Prof. Zhao) on reasonable request.

\section{Competing interests}

The authors declare that they have no known competing interests that could have appeared to influence the work reported in this paper.

\section{Funding}

This work was supported by the National Science and Technology Major Project (No. 2018ZX10103001) and the Chinese Center for Disease Control and Prevention Project (59911916).

\section{Authors' contributions}

Chunfa Liu: Data collection, Data analysis, Writing-Original draft preparation; Ping He: Methodology, Software; Jiale Fan: Data collection, Perform experiments; Aijing Ma: Data collection, Perform experiments; Wencong He: Data collection, Perform experiments; Bing Zhao: Data collection, Perform experiments; Shengfen Wang: Data collection; Guangxue He: Supervision; Yanlin Zhao:

Conceptualization, Study design and Writing-Reviewing and Editing. All authors reviewed the manuscript.

\section{Acknowledgements}

All the staff from National Tuberculosis Reference Laboratory in China is also highly acknowledged.

\section{References}

1. WHO, Globle tuberculosis report 2020. Geneva: World Health Organization, 2020. https://www.who.int/teams/global-tuberculosis-programme/tb-reports.

2. Yang, C. and Q. Gao, Recent transmission of Mycobacterium tuberculosis in China: the implication of molecular epidemiology for tuberculosis control. Frontiers of medicine, 2018. 12(1): 76-83.

3. Nsofor, C.A., Q. Jiang, J. Wu, M. Gan, Q. Liu, T. Zuo, et al., Transmission is a Noticeable Cause of Resistance Among Treated Tuberculosis Patients in Shanghai, China. Scientific reports, 2017. 7(1): 7691.

4. Houben, R., N. Menzies, T. Sumner, G. Huynh, N. Arinaminpathy, J. Goldhaber-Fiebert, et al., Feasibility of achieving the 2025 WHO global tuberculosis targets in South Africa, China, and India: a combined analysis of 11 mathematical models. The Lancet. Global health, 2016. 4(11): e806-e15.

5. Kohl, T.A., D. Harmsen, J. Rothganger, T. Walker, R. Diel, and S. Niemann, Harmonized Genome Wide Typing of Tubercle Bacilli Using a Web-Based Gene-By-Gene Nomenclature System. EBioMedicine, 2018. 34: 131-8. 
6. Yang, C., T. Luo, X. Shen, J. Wu, M. Gan, P. Xu, et al., Transmission of multidrug-resistant Mycobacterium tuberculosis in Shanghai, China: a retrospective observational study using wholegenome sequencing and epidemiological investigation. The Lancet Infectious Diseases, 2017. 17(3): 275-84.

7. Roetzer, A., R. Diel, T. Kohl, C. Rückert, U. Nübel, J. Blom, et al., Whole genome sequencing versus traditional genotyping for investigation of a Mycobacterium tuberculosis outbreak: a longitudinal molecular epidemiological study. PLoS medicine, 2013. 10(2): e1001387.

8. Lalor, M., N. Casali, T. Walker, L. Anderson, J. Davidson, N. Ratna, et al., The use of whole-genome sequencing in cluster investigation of a multidrug-resistant tuberculosis outbreak. The European respiratory journal, 2018. 51(6).

9. Gardy, J., J. Johnston, S. Ho Sui, V. Cook, L. Shah, E. Brodkin, et al., Whole-genome sequencing and social-network analysis of a tuberculosis outbreak. The New England journal of medicine, 2011. 364(8): 730-9.

10. Kohl, T., D. Harmsen, J. Rothgänger, T. Walker, R. Diel, and S. Niemann, Harmonized Genome Wide Typing of Tubercle Bacilli Using a Web-Based Gene-By-Gene Nomenclature System. EBioMedicine, 2018. 34: 131-8.

11. Cirillo, D.M., A.M. Cabibbe, M.R. De Filippo, A. Trovato, T. Simonetti, G.M. Rossolini, et al., Use of WGS in Mycobacterium tuberculosis routine diagnosis. International journal of mycobacteriology, 2016. 5 Suppl 1: S252-S3.

12. Jajou, R., T.A. Kohl, T. Walker, A. Norman, D.M. Cirillo, E. Tagliani, et al., Towards standardisation: comparison of five whole genome sequencing (WGS) analysis pipelines for detection of epidemiologically linked tuberculosis cases. Euro surveillance: bulletin Europeen sur les maladies transmissibles = European communicable disease bulletin, 2019. 24(50).

13. Kohl, T.A., R. Diel, D. Harmsen, J. Rothganger, K.M. Walter, M. Merker, et al., Whole-Genome-Based Mycobacterium tuberculosis Surveillance: a Standardized, Portable, and Expandable Approach. Journal of Clinical Microbiology, 2014. 52(7): 2479-86.

14. Yang, C., L. Lu, J.L. Warren, J. Wu, Q. Jiang, T. Zuo, et al., Internal migration and transmission dynamics of tuberculosis in Shanghai, China: an epidemiological, spatial, genomic analysis. The Lancet Infectious Diseases, 2018. 18(7): 788-95.

15. Jiang, Q., Q. Liu, L. Ji, J. Li, Y. Zeng, L. Meng, et al., Citywide Transmission of Multidrug-resistant Tuberculosis Under China's Rapid Urbanization: A Retrospective Population-based Genomic Spatial Epidemiological Study. Clinical infectious diseases: an official publication of the Infectious Diseases Society of America, 2020. 71(1): 142-51.

16. Zhao, Y., S. Xu, L. Wang, D. Chin, S. Wang, G. Jiang, et al., National survey of drug-resistant tuberculosis in China. The New England journal of medicine, 2012. 366(23): 2161-70.

17. Phelan, J.E., D.M. O'Sullivan, D. Machado, J. Ramos, Y.E.A. Oppong, S. Campino, et al., Integrating informatics tools and portable sequencing technology for rapid detection of resistance to antituberculous drugs. Genome Medicine, 2019. 11(1): 41. 
18. Hunt, M., P. Bradley, S.G. Lapierre, S. Heys, M. Thomsit, M.B. Hall, et al., Antibiotic resistance prediction for Mycobacterium tuberculosis from genome sequence data with Mykrobe. Wellcome open research, 2019. 4: 191.

19. Walker, T.M., P. Monk, E.G. Smith, and T.E. Peto, Contact investigations for outbreaks of Mycobacterium tuberculosis: advances through whole genome sequencing. Clinical microbiology and infection: the official publication of the European Society of Clinical Microbiology and Infectious Diseases, 2013. 19(9): 796-802.

20. Andreas Roetzer, R.D., Thomas A. Kohl1, Christian Ru Jo ckert, Ulrich Nu Thierry Wirth, Sebastian Jaenicke, Sieglinde Schuback, Sabine Rurn Kalinowski, Stefan Niemann, Whole Genome Sequencing versus Traditional Genotyping for Investigation of a Mycobactrium tuberculosis Outbreak: A Longitudinal Molecular Epidemiological Study. PLoS medicine, 2013. 10(2): e1001387.

21. Meehan, C.J., P. Moris, T.A. Kohl, J. Pecerska, S. Akter, M. Merker, et al., The relationship between transmission time and clustering methods in Mycobacterium tuberculosis epidemiology. EBioMedicine, 2018. 37: 410-6.

22. Nikolayevskyy, V., S. Niemann, R. Anthony, D. van Soolingen, E. Tagliani, C. Kodmon, et al., Role and value of whole genome sequencing in studying tuberculosis transmission. Clinical microbiology and infection: the official publication of the European Society of Clinical Microbiology and Infectious Diseases, 2019. 25(11): 1377-82.

23. Jones, R.C., L.G. Harris, S. Morgan, M.C. Ruddy, M. Perry, R. Williams, et al., Phylogenetic Analysis of Mycobacterium tuberculosis Strains in Wales by Use of Core Genome Multilocus Sequence Typing To Analyze Whole-Genome Sequencing Data. J Clin Microbiol, 2019. 57(6).

24. Nikolayevskyy, V., K. Kranzer, S. Niemann, and F. Drobniewski, Whole genome sequencing of Mycobacterium tuberculosis for detection of recent transmission and tracing outbreaks: $A$ systematic review. Tuberculosis (Edinb), 2016. 98: 77-85.

25. Luo, T., C. Yang, Y. Peng, L. Lu, G. Sun, J. Wu, et al., Whole-genome sequencing to detect recent transmission of Mycobacterium tuberculosis in settings with a high burden of tuberculosis. Tuberculosis (Edinb), 2014. 94(4): 434-40.

26. Jiang, Q., Q. Liu, L. Ji, J. Li, Y. Zeng, L. Meng, et al., Citywide Transmission of Multidrug-resistant Tuberculosis Under China's Rapid Urbanization: A Retrospective Population-based Genomic Spatial Epidemiological Study. Clinical infectious diseases: an official publication of the Infectious Diseases Society of America, 2020. 71(1): 142-51.

27. Feng, M., Y. Xu, X. Zhang, Q. Qiu, S. Lei, J. Li, et al., Risk factors of multidrug-resistant tuberculosis in China: A meta-analysis. Public health nursing, 2019. 36(3): 257-69.

28. Cai, X., D. Zhang, Y. Yan, D. Tan, and Y. Xu, Meta-analysis on risk factors of multidrug resistant tuberculosis in China. Zhonghua liuxingbingxue zazhi, 2015. 36(12): 1424-9.

29. Shi, W., L. Davies Forsman, Y. Hu, X. Zheng, Y. Gao, X. Li, et al., Improved treatment outcome of multidrug-resistant tuberculosis with the use of a rapid molecular test to detect drug resistance in 
China. International journal of infectious diseases: IJID : official publication of the International Society for Infectious Diseases, 2020. 96: 390-7.

30. Jajou, R., T. van der Laan, R. de Zwaan, M. Kamst, A. Mulder, A. de Neeling, et al., WGS more accurately predicts susceptibility of Mycobacterium tuberculosis to first-line drugs than phenotypic testing. Journal of Antimicrobial Chemotherapy, 2019. 74(9): 2605-16.

31. Moreno-Molina, M., I. Comas, and V. Furió, The Future of TB Resistance Diagnosis: The Essentials on Whole Genome Sequencing and Rapid Testing Methods. Archivos de bronconeumologia, 2019. 55(8): 421-6

\section{Figures}

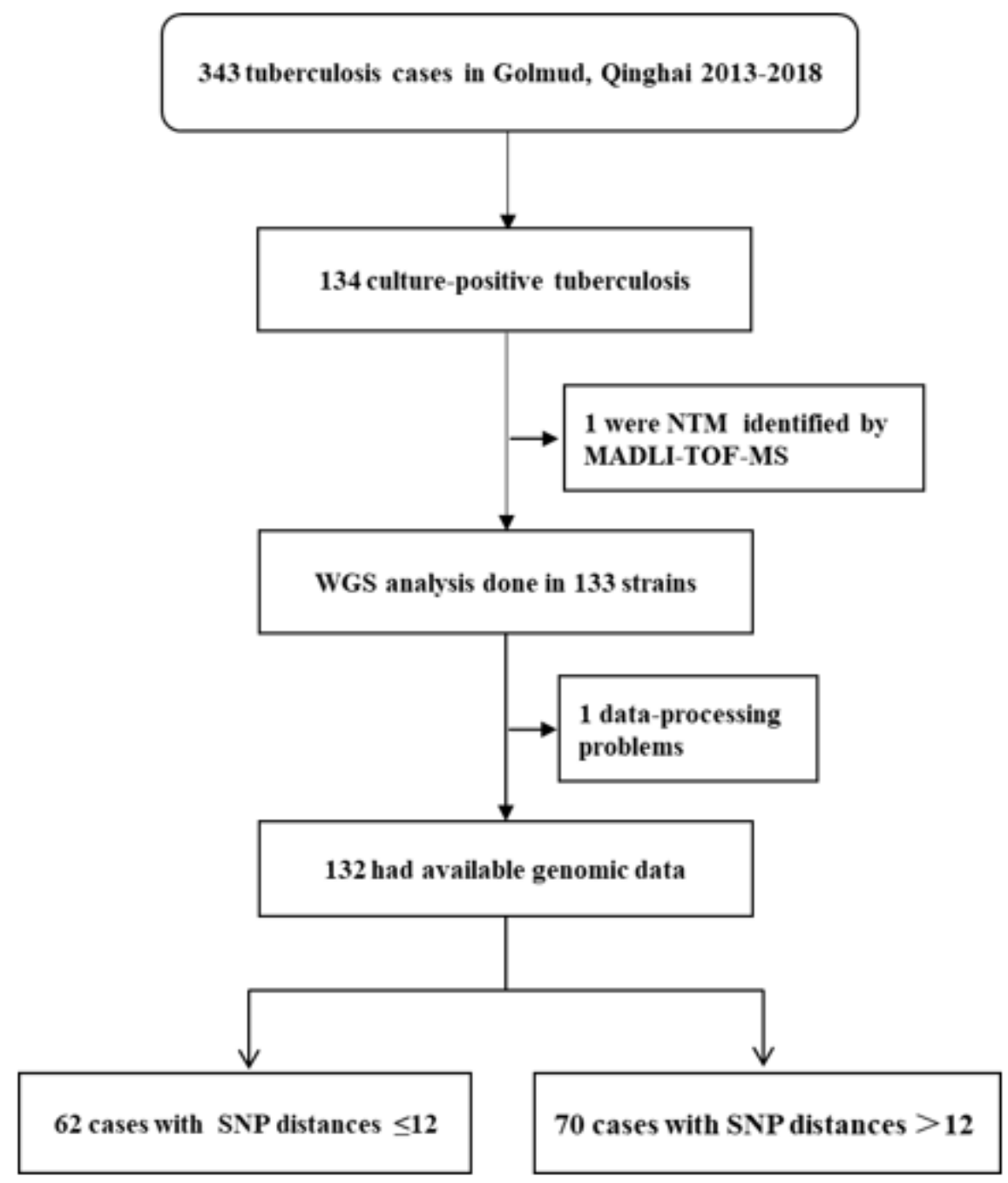

\section{Figure 1}

Diagram of cluster analysis using WGS 
A

Drug resistant rate

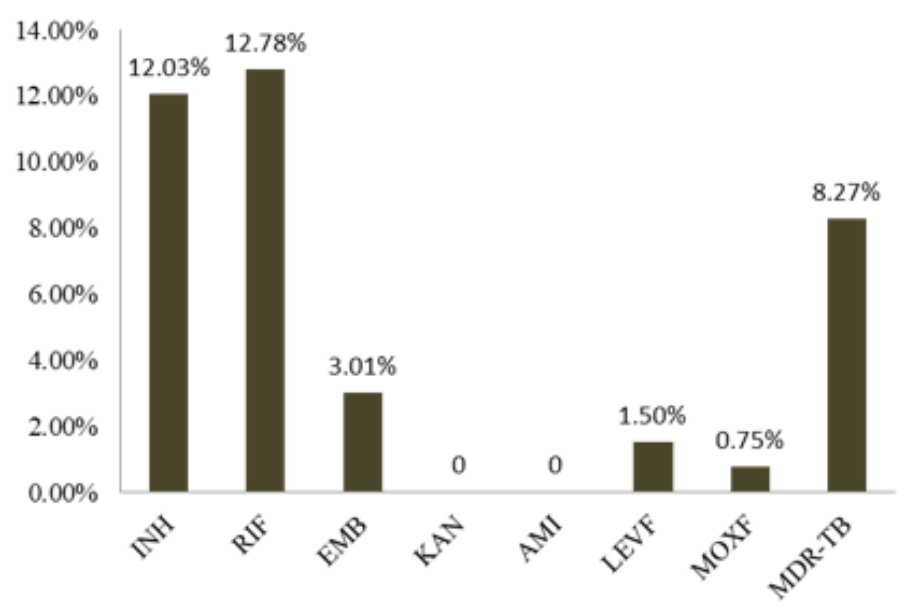

B

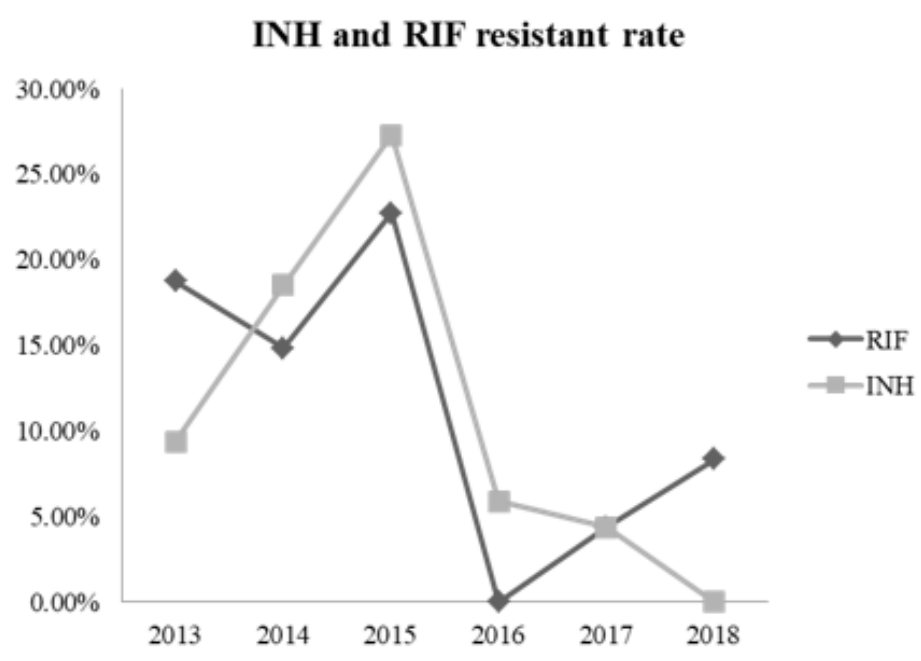

Figure 2

Phenotypic drug susceptibility test results. A. The drug resistant rate of different antibiotics; B. INH and RIF resistant rates with years 
A

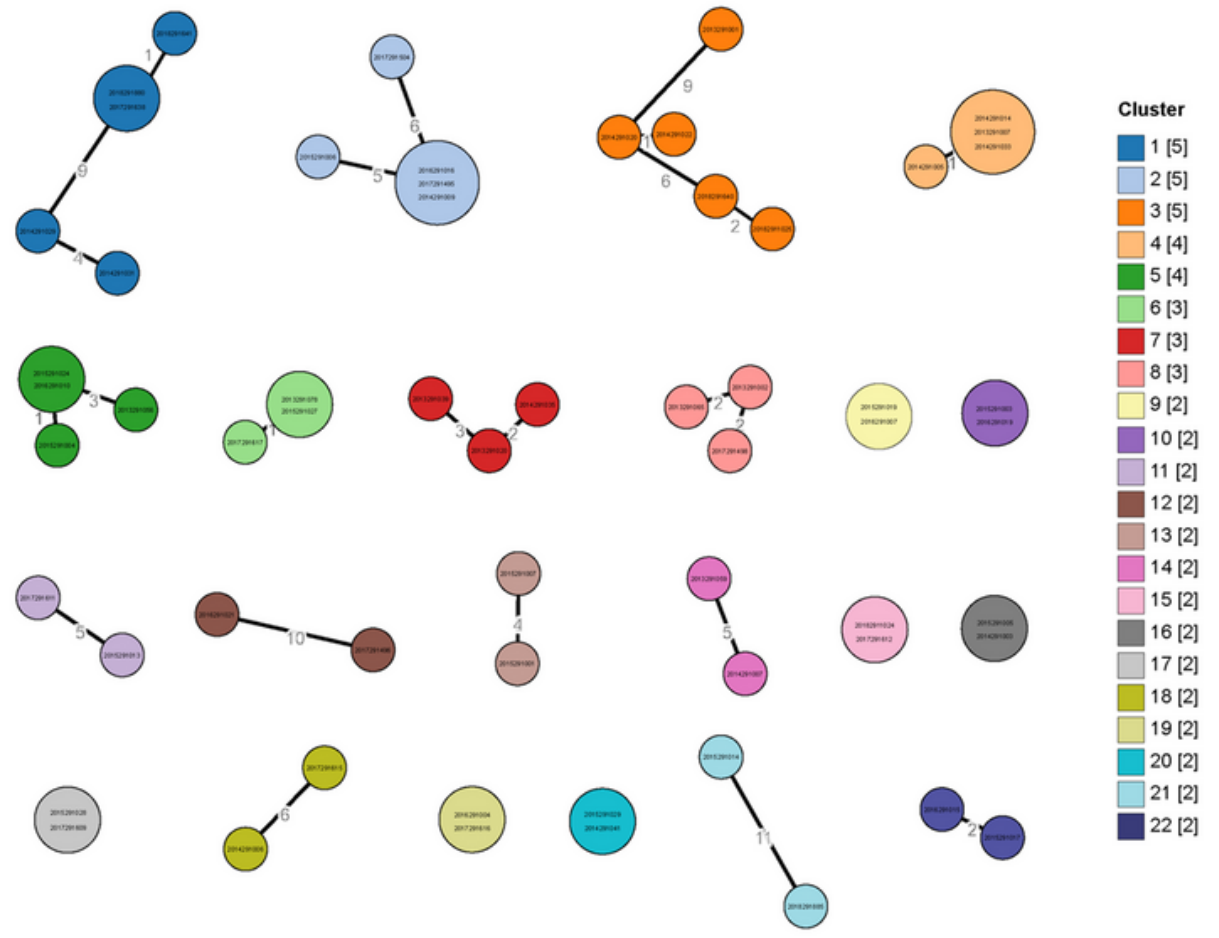

B
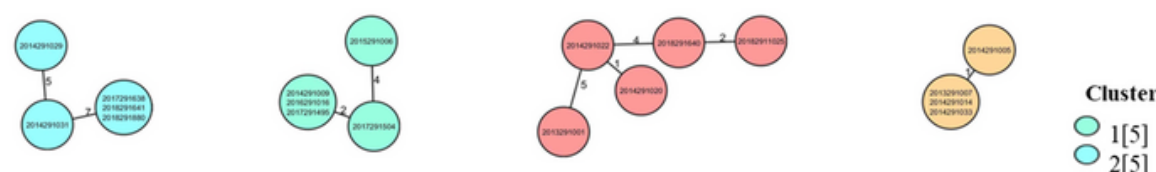

1[5]

3[5]
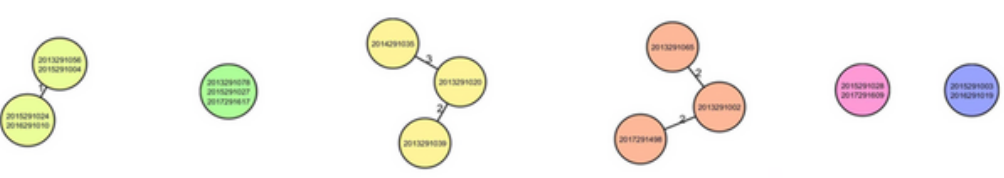

단]

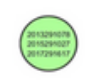

6[3]

$7[3]$

$\bigcirc 9[2]$

10[2]

11[2]

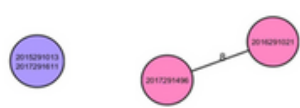

(

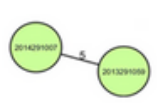

$\because$

$\mathrm{O}_{13[2]}$

14[2]

․ 15[2]

16[2]

17[2]

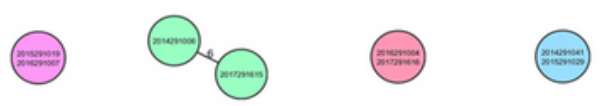

$\infty$

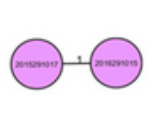

19[2]

○ 20[2]

○ 21[2]

22[2]

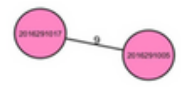

Figure 3

Minimum distance spanning tree (MST) of strains with 12 or fewer SNPs. A. cg-MLST analysis; B. SNPbased approach 


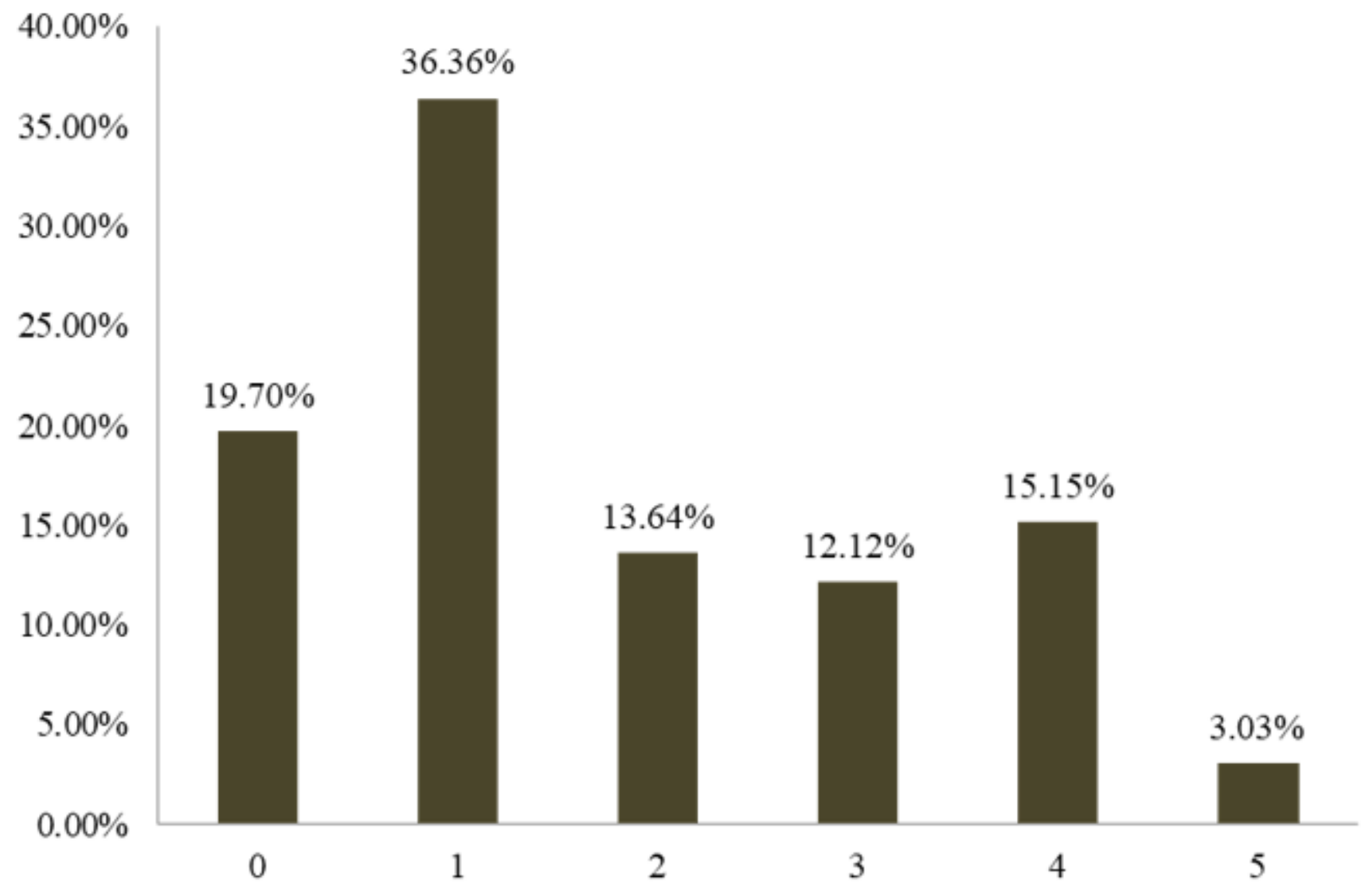

Figure 4

Pairs based on diagnosis interval (year) in all clusters 


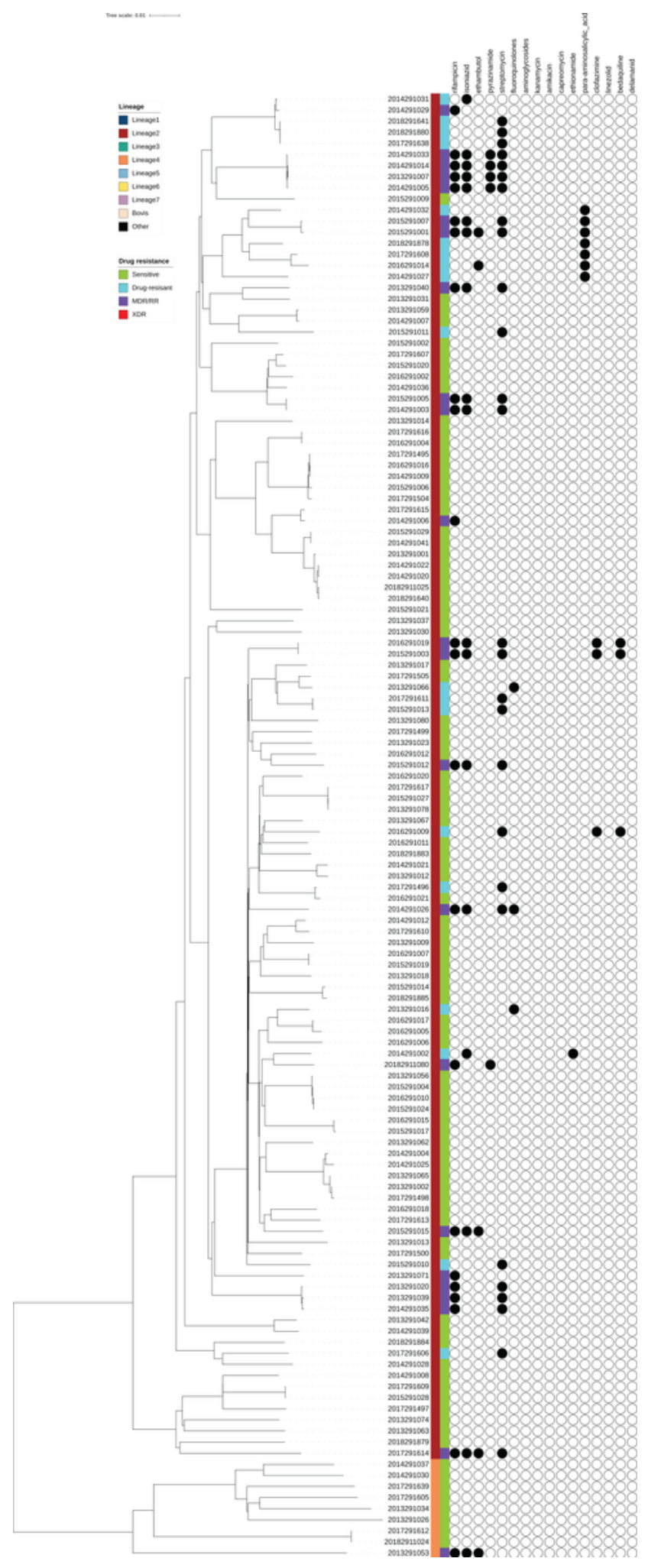

Figure 5

ML Phylogenetic tree for $132 \mathrm{Mtb}$ strains 
A

B

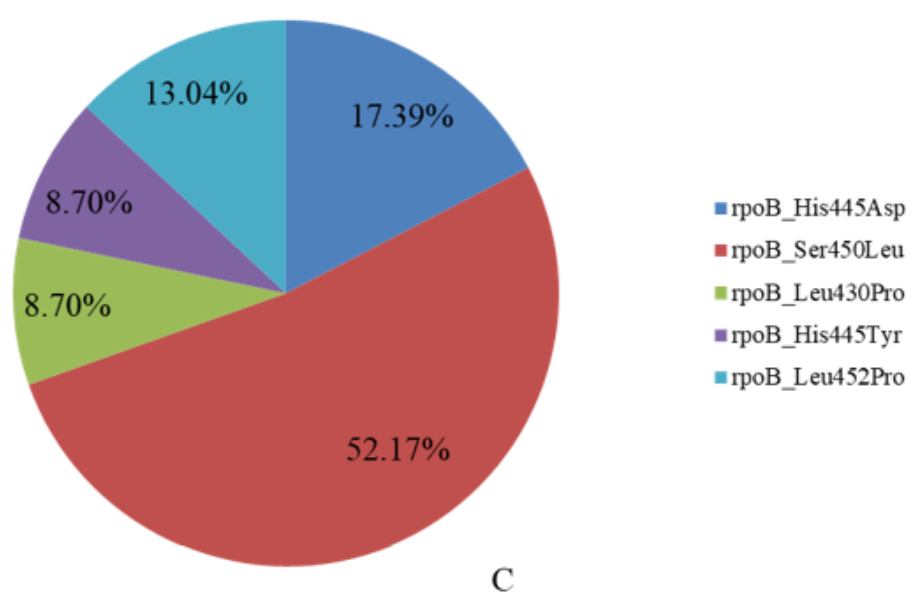

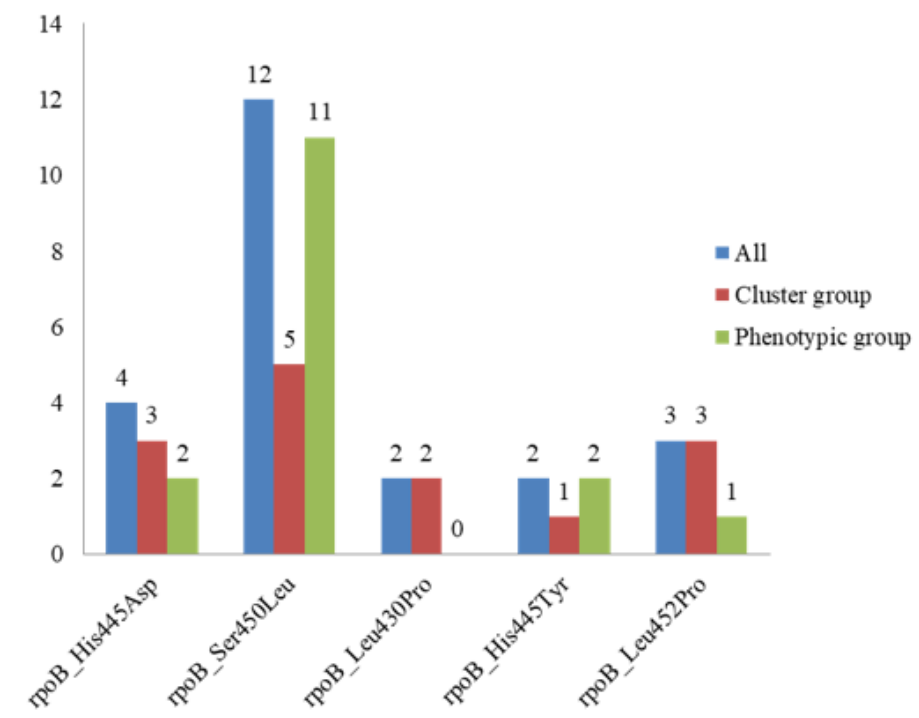

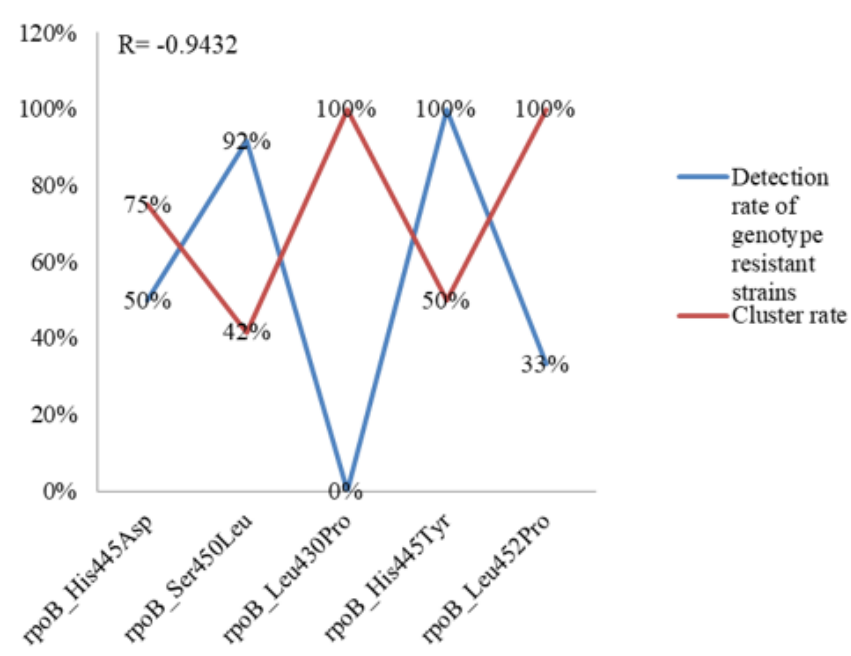

Figure 6

Resistant mutation sites of RIF phenotypic resistant strains. A. Resistant mutation sites distribution among 23 RIF genotypic resistant strains; B. The strains number in Cluster and phenotypic groups based on RIF resistant associated mutation sites. C. The correlation between cluster rate and phenotypic resistant detection rate among all 23 RIF genotypic resistant strains based on each mutation site $(\mathrm{R}=-0.9432)$. 